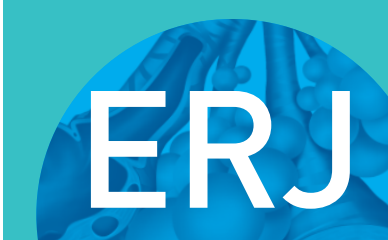

open research

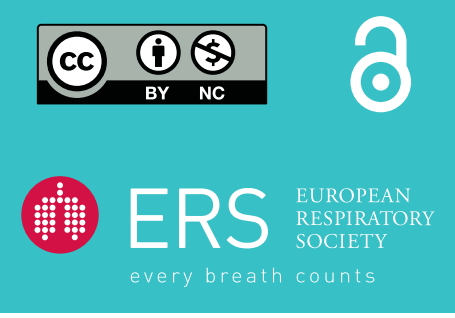

\section{The course of specific self-reported exercise-induced airway symptoms in adolescents with and without asthma}

\author{
To the Editor:
}

Airway symptoms in conjunction with exercise can take on many forms and can have several contributory factors such as exercise-induced bronchoconstriction (EIB), exercise-induced laryngeal obstruction or hyperventilation [1]. Previous longitudinal studies on self-reported exercise-induced airway symptoms among adolescents have primarily studied single symptoms, focusing on dyspnoea or wheeze, often as a way to investigate the prevalence of asthma [2,3]. To the best of our knowledge, there are, as yet, no population-based studies investigating the difference between adolescents with and without current asthma regarding the development of different specific exercise-induced airway symptoms. Therefore, we studied the natural course of self-reported exercise-induced dyspnoea, throat tightness, wheeze, chest tightness, cough, stridor and hoarseness over a 5-year period among adolescents with and without current asthma.

The ANDAS study is a prospective questionnaire-based cohort study of exercise-induced airway symptoms, asthma and physical activity among adolescents in a general population from Uppsala, Sweden [4]. At baseline in 2011, all adolescents, aged 12-13 years, in the city of Uppsala ( $n=3838$ ) were contacted by post and invited to complete a web-based questionnaire. In total, $60 \%$ of the adolescents responded. 5 years later, in 2016, adolescents who had responded to the questionnaire in 2011 (now 17-18 years old) were invited to complete the same questionnaire again. The response rate was $43 \%(n=1002)$. The recruitment procedure and the characteristics of participants and nonparticipants have been described previously [5].

In the present study, two specified subpopulations from the ANDAS study were analysed: participants reporting current asthma both in 2011 and 2016 ( $n=55$; mean age 2011: 129 years; 2016: 17.6 years; 60\% females) and participants without asthma at both time points ( $n=844$; mean age 2011: 12.7 years; 2016 : 17.4 years; $56 \%$ females). 36 adolescents reporting asthma at baseline but not at follow-up and 67 adolescents reporting asthma only at follow up were excluded from further analyses. Exercise-induced airway symptoms were defined as reporting any of the following symptoms during or directly after exercise in the past year: dyspnoea, throat tightness, wheeze, chest tightness, cough, stridor and hoarseness. Current asthma was defined as ever having been diagnosed with asthma by a physician and having used asthma medication and/ or having had asthma symptoms (wheeze, or daytime or nocturnal dyspnoea) in the last 12 months. Level of physical activity was defined as number of days per week with $\geqslant 60 \mathrm{~min}$ of physical activity [6].

The data were analysed using STATA 14.2 (StataCorp, College Station, TX, USA). Differences in percentages were calculated using a two-sided $\chi^{2}$-test or Fisher's exact test between groups and McNemar test for within-group comparisons. The Student's t-test was used for comparisons between groups regarding physical activity.

Among adolescents with current asthma, the prevalence rates of all the specific exercise-induced symptoms were similar at baseline and follow-up. Exercise-induced dyspnoea, throat tightness and wheeze were the most prevalent symptoms at both baseline and follow-up (figure 1). In contrast, in the group without

@ERSpublications

In a general population, the prevalence of exercise-induced cough, dyspnoea, throat and chest tightness, wheeze, and stridor increases from adolescence to young adulthood among individuals without asthma in contrast to individuals with asthma https://bit.ly/3hR57OX

Cite this article as: Johansson H, Emtner M, Janson C, et al. The course of specific self-reported exercise-induced airway symptoms in adolescents with and without asthma. ERJ Open Res 2020; 6: 00349-2020 [https://doi.org/10.1183/23120541.00349-2020].

Copyright $\odot$ ERS 2020. This article is open access and distributed under the terms of the Creative Commons Attribution NonCommercial Licence 4.0. 


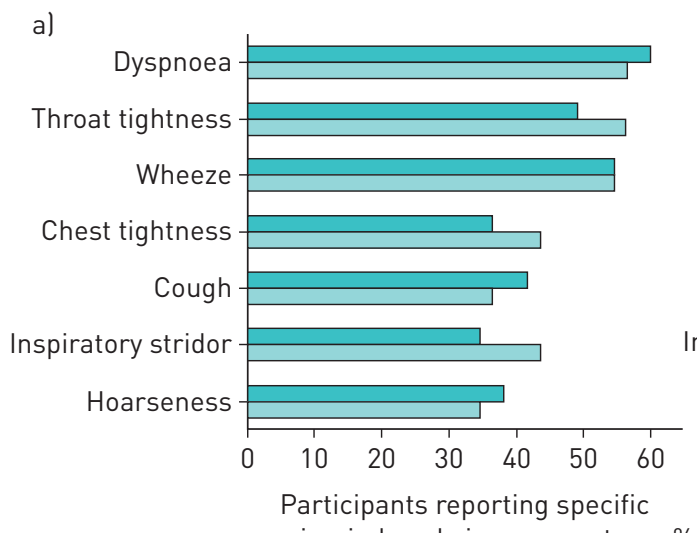

exercise-induced airway symptoms \%

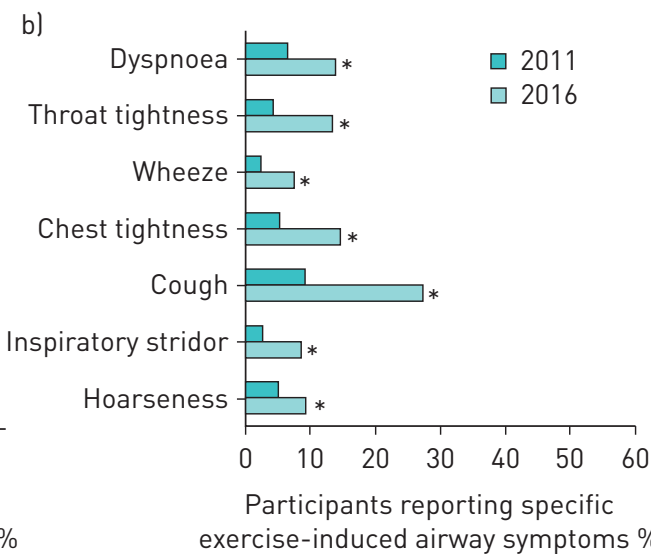

FIGURE 1 Percentage of participants al with and b) without current asthma reporting specific exercise-induced airway symptom at baseline in 2011 and at follow-up in 2016. *: p<0.05 for change in prevalence of symptoms from baseline to follow-up.

current asthma, the prevalence of all the specific exercise-induced airway symptoms increased significantly with age. Exercise-induced cough was the most common symptom at both time points (figure 1).

Among adolescents with asthma, the prevalence of rhinitis was $60 \%$ in 2011 and $63 \%$ in 2016. The corresponding figures for the non-asthma group were $13 \%$ and $25 \%$. The prevalence of overweight and obesity was $26 \%$ in 2011 and $20 \%$ in 2016 among those with asthma. The corresponding figures for the non-asthma group were $19 \%$ and $14 \%$, respectively.

The level of self-reported physical activity decreased in both groups with age but did not differ between participants with asthma compared to participants without asthma (baseline: 4.3 versus 4.1 days, $\mathrm{p}=0.46$; follow-up: 3.5 versus 3.6 days, $\mathrm{p}=0.97)$.

At baseline, among participants with asthma, more females reported exercise-induced wheeze and inspiratory stridor compared with males ( $75 \%$ versus $25 \% \mathrm{p}<0.001 ; 45$ versus $18 \%, \mathrm{p}=0.03$, respectively). At follow up, females reported higher prevalence of exercise-induced throat tightness and inspiratory stridor compared with males (75\% versus $24 \%, \mathrm{p}<0.001 ; 74 \%$ versus $26 \%, \mathrm{p}<0.001$, respectively). More females than males also reported exercise-induced dyspnoea, wheeze, chest tightness and hoarseness $(p<0.05)$. In addition, in the group without asthma, females had a higher prevalence of exercise-induced dyspnoea, chest tightness, inspiratory stridor and throat tightness at baseline $(\mathrm{p}<0.05)$, and all symptoms at follow-up $(\mathrm{p}<0.05)$, except for cough and hoarseness.

In the group without current asthma, the rate of remission of symptoms decreased with increasing number of exercise-induced symptoms reported at baseline. Adolescents reporting three or more exercise-induced symptoms at baseline had a significantly lower remission rate compared with adolescents reporting one symptom $(21.2 \%$ versus $51.7 \%, \mathrm{p}=0.004)$ and two symptoms $(21.2 \%$ versus $46.8 \%, \mathrm{p}=0.03)$.

Among adolescents without asthma, we found a significant increase in the prevalence of all specific exercise-induced airway symptoms over a 5-year period. Previous studies, investigating single symptoms, have reported increased prevalence of cough and wheeze with age [7, 8]. The prevalence of exercise-induced dyspnoea, the second most common symptom in the group without asthma in the present study, was more than double at follow-up. Although exercise-induced dyspnoea can be related to EIB [9], several studies have shown dyspnoea to be a poor predictor of EIB [10-12]. Several other conditions can cause dyspnoea during exercise, deconditioning being reported to be the most common one $[11,13]$.

Among participants with current asthma, the prevalence of symptoms did not increase with age. Four out of five participants with asthma reported exercise-induced airway symptoms, in line with a previous cross-sectional study [14].

Within the asthma group, exercise-induced dyspnoea and throat tightness were the two most common exercise-related symptoms. Exercise-induced cough was the most common symptom in the non-asthma group. Symptoms usually associated with disorders in the larynx or upper airways were far more prevalent among females than males, both in the asthma and the non-asthma group. Indeed, in females with current 
asthma, almost two thirds reported exercise-induced throat tightness and almost half reported inspiratory stridor. To our knowledge, this has not previously been described among adolescents with asthma. The clinical significance of these findings needs to be further investigated.

A larger proportion of adolescents reporting three or more exercise-induced symptoms at baseline were found to have persistent symptoms at follow-up, compared with those with fewer symptoms. This may imply that a clinical history, covering on a wide range of symptoms, might better identify who is likely to have persistent exercise-induced airway symptoms.

The strength of the present study is the relatively large unselected sample of adolescents from the general population investigated, that it encompassed a wide range of questions regarding exercise-induced symptoms and its longitudinal design. Therefore, this study adds information to the limited data available on the course of specific exercise-induced airway symptoms from adolescence to young adulthood. The results should, however, be interpreted with caution, as symptoms, in the absence of objective tests, lack diagnostic sensitivity and specificity. To draw a firm conclusion, future studies of larger populations and in different geographical locations are needed. The response rate dropped from $60 \%$ to $43 \%$ from baseline to follow-up, implicating a possible responder bias. However, the nonresponders did not differ significantly from responders according to baseline data, suggesting that the cohort studied at follow-up was representative of that studied at baseline [5].

In young subjects without asthma, the prevalence of exercise-induced throat tightness, inspiratory stridor, cough, chest tightness, wheeze and dyspnoea increased with age from adolescence to young adulthood. In contrast, among subjects with asthma, the prevalence of individual respiratory symptoms was consistent over time. Nonasthmatic subjects reporting multiple exercise-induced symptoms during adolescence are less likely to report remission of symptoms 5 years later.

\section{Henrik Johansson $\oplus^{1,2,3}$, Margareta Emtner ${ }^{2}$, Christer Janson $\oplus^{2}$, Leif Nordang $^{4}$ and Andrei Malinovschi $\oplus^{1}$ \\ ${ }^{1}$ Dept of Medical Sciences, Clinical Physiology, Uppsala University, Uppsala, Sweden. ${ }^{2}$ Dept of Medical Sciences, Respiratory, Allergy and Sleep Research, Uppsala University, Uppsala, Sweden. ${ }^{3}$ Dept of Neuroscience, Physiotherapy, Uppsala University, Uppsala, Sweden. ${ }^{4}$ Dept of Surgical Sciences, Otorhinolaryngology, and Head and Neck Surgery, Uppsala University, Uppsala, Sweden.}

Correspondence: Henrik Johansson, Fysioterapi, Institutionen för Neurovetenskap, Uppsala universitet, Box 593 BMC, 75124 Uppsala, Sweden. E-mail: henrik.johansson@neuro.uu.se

Received: 6 June 2020 | Accepted after revision: 14 Sept 2020

Conflict of interest: None declared.

Support statement: This work was supported by The Signhild Engkvist Foundation and Bror Hjerpstedts Foundation, Sweden. Funding information for this article has been deposited with the Crossref Funder Registry.

\section{References}

1 Weatherald J, Lougheed MD, Taillé C, et al. Mechanisms, measurement and management of exertional dyspnoea in asthma. Eur Respir Rev 2017; 26: 170015.

2 Pearce N, Aït-Khaled N, Beasley R, et al. Worldwide trends in the prevalence of asthma symptoms: phase III of the International Study of Asthma and Allergies in Childhood (ISAAC). Thorax 2007; 62: 758-766.

3 Hong SJ, Lee MS, Sohn MH, et al. Self-reported prevalence and risk factors of asthma among Korean adolescents: 5-year follow-up study, 1995-2000. Clin Exp Allergy 2004; 34: 1556-1562.

4 Johansson $\mathrm{H}$, Norlander $\mathrm{K}$, Hedenstrom $\mathrm{H}$, et al. Exercise-induced dyspnea is a problem among the general adolescent population. Respir Med 2014; 108: 852-858.

5 Johansson $\mathrm{H}$, Norlander K, Malinovschi A. Increased prevalence of exercise-induced airway symptoms - a five-year follow-up from adolescence to young adulthood. Respir Med 2019; 154: 76-81.

6 Currie C, Nic Gabhainn S, Godeau E, et al. The Health Behaviour in School-aged Children: WHO Collaborative Cross-National (HBSC) study: origins, concept, history and development 1982-2008. Int J Public Health 2009; 54 Suppl 2: 131-139.

7 Jurca M, Ramette A, Dogaru CM, et al. Prevalence of cough throughout childhood: a cohort study. PLoS One 2017; 12: e0177485.

8 Jurca M, Pescatore AM, Goutaki M, et al. Age-related changes in childhood wheezing characteristics: a whole population study. Pediatr Pulmonol 2017; 52: 1250-1259.

9 Melani AS, Ciarleglio G, Pirrelli M, et al. Perception of dyspnea during exercise-induced bronchoconstriction. Respir Med 2003; 97: 221-227.

10 Johansson $\mathrm{H}$, Norlander K, Berglund L, et al. Prevalence of exercise-induced bronchoconstriction and exercise-induced laryngeal obstruction in a general adolescent population. Thorax 2015; 70: 57-63.

11 Seear M, Wensley D, West N. How accurate is the diagnosis of exercise induced asthma among Vancouver schoolchildren? Arch Dis Child 2005; 90: 898-902. 
12 De Baets F, Bodart E, Dramaix-Wilmet M, et al. Exercise-induced respiratory symptoms are poor predictors of bronchoconstriction. Pediatr Pulmonol 2005; 39: 301-305.

13 Abu-Hasan M, Tannous B, Weinberger M. Exercise-induced dyspnea in children and adolescents: if not asthma then what? Ann Allergy Asthma Immunol 2005; 94: 366-371.

14 Ostrom NK, Eid NS, Craig TJ, et al. Exercise-induced bronchospasm in children with asthma in the United States: results from the Exercise-Induced Bronchospasm Landmark Survey. Allergy Asthma Proc 2011; 32: 425-430. 